\title{
Administrative risk quantification of subcutaneous and intravenous therapies in Italian centers utilizing the Failure Mode and Effects Analysis approach
}

\author{
This article was published in the following Dove Press journal: \\ ClinicoEconomics and Outcomes Research \\ 3 August 2016 \\ Number of times this article has been viewed
}

\author{
Clemente Ponzetti' \\ Monica Canciani² \\ Massimo Farina ${ }^{2}$ \\ Sara $\mathrm{Era}^{3}$ \\ Stefan Walzer ${ }^{4,5}$ \\ 'Gruppo Policlinico di Monza, \\ Alessandria, ANMDO National \\ Association of Hospital Physicians, \\ Bologna, ${ }^{2}$ Studio EmmEffe Srl, Milan, \\ ${ }^{3}$ Roche Spa, Monza, Italy; ${ }^{4}$ MArS \\ Market Access \& Pricing Strategy \\ GmbH, Weil am Rhein, ${ }^{5}$ State \\ University Baden-Wuerttemberg, \\ Health Care Management, Loerrach, \\ Germany
}

Background: In oncology, an important parameter of safety is the potential treatment error in hospitals. The analyzed hypothesis is that of subcutaneous therapies would provide a superior safety benefit over intravenous therapies through fixed-dose administrations, when analyzed with trastuzumab and rituximab.

Methods: For the calculation of risk levels, the Failure Mode and Effect Analysis approach was applied. Within this approach, the critical treatment path is followed and risk classification for each individual step is estimated. For oncology and hematology administration, 35 different risk steps were assessed. The study was executed in 17 hematology and 16 breast cancer centers in Italy. As intravenous and subcutaneous were the only injection routes in medical available for trastuzumab and rituximab in oncology at the time of the study, these two therapies were chosen.

Results: When the risk classes were calculated, eight high-risk areas were identified for the administration of an intravenous therapy in hematology or oncology; 13 areas would be defined as having a median-risk classification and 14 areas as having a low-risk classification (total risk areas: $\mathrm{n}=35$ ). When the new subcutaneous formulation would be applied, 23 different risk levels could be completely eliminated (65\% reduction). Important high-risk classes such as dose calculation, preparation and package labeling, preparation of the access to the vein, pump infusion preparation, and infusion monitoring were included in the eliminations. The overall risk level for the intravenous administration was estimated to be 756 (ex-ante) and could be reduced by $70 \%$ (ex-post). The potential harm compensation for errors related to pharmacy would be decreased from eight risk classes to only three risk classes.

Conclusion: The subcutaneous administration of trastuzumab (breast cancer) and rituximab (hematology) might lower the risk of administration and treatment errors for patients and could hence indirectly have a positive financial impact for hospitals.

Keywords: health economics, safety, insurance premium, oncology, intravenous therapy, subcutaneous therapy

\section{Introduction}

Oncology includes a variety of different diseases and indications and can hardly be seen as one disease on its own. On the basis of the therapy advances in most recent years and the severity of the malignancy, the overall survival ranges from a few months
Studio EmmEffe Srl, Via Messina 43, Milan 20154, Italy

Email mfarina@mfsrl.it 
to many years in the metastatic setting. In adjuvant indications where tumors are being detected at an early stage, cure could also be achieved. ${ }^{1}$

The goals of cancer care are to optimize both the length and quality of life, ${ }^{2,3}$ which could be achieved with different treatment options. In recent years innovative treatments have been introduced in oncology and hematology.

Oncology medications as monotherapy and combinations have an acceptable risk-benefit profile; however, they are also linked with more or less patient-relevant side effects. ${ }^{4}$ The AIFA (Agenzia Italiana del Farmaco, Italian Medicine Agency) definition of side effects is as follows: harmful and unwanted event resulting from the use of a medicinal product. In fact with this definition, regardless of the use of the medicine, adverse reactions will be reported, including those arising from medication error, abuse, against misuse, off-label, overdose, and professional exposure. ${ }^{5}$ The adverse reactions are mainly related to the mode of action of these therapies. Another important parameter of safety is the potential treatment error, which could occur in hospitals and might have an even bigger impact on adverse events due to a noncompliant and nonlabeled dosing. ${ }^{6}$ Such errors could be assumed to occur more often with difficult dosing regimens and generally with therapies for which therapy dosing per patient (eg, per kg or per $\mathrm{m}^{2}$ ) is administered. Furthermore, errors could occur with oral medications in case these therapies are not taken at all or are administered to the wrong persons. Generally, such mistakes might occur more often with intravenous therapies. Such treatment errors can impact patients' quality of life and sometimes survival. ${ }^{4,6}$ Additionally, hospitals need to cover their liability with adequate insurances. The higher the risk for such treatment errors, the higher the insurance premium a hospital needs to pay annually. If a hospital would choose not to pay premiums, they would need to accrue higher amounts in their accounting for the potential financial implications of such an error.

Subcutaneous versions of rituximab and trastuzumab have been available since 2014. ${ }^{1,7}$ Subcutaneous therapy should benefit all stakeholders in the health care system, especially if these are delivered as fixed-dose regimens. The analyzed hypothesis is a superior safety benefit of subcutaneous over intravenous therapies through fixed-dose administrations exemplary analyzed with trastuzumab and rituximab. As intravenous and subcutaneous were the only injection routes in medical administration available for trastuzumab and rituximab in oncology at the time of the study, these two therapies were chosen. The purpose of the underlying study was to analyze the risk impact of a subcutaneous therapy in comparison to an intravenous therapy in an Italian health care setting focusing on breast cancer and NHL with trastuzumab and rituximab, respectively.

\section{Methods}

In order to analyze the impact of a subcutaneous administration of an existing therapy in comparison to the intravenous mode, a primary research study in Italy was executed. The primary objectives of the study were to quantify the risk and cost implications from different perspectives (patients, hospital administration, and medical staff) using the Failure Mode and Effect Analysis (FMEA) approach. ${ }^{8}$ FMEA was developed outside of health care and is now being used in health care to assess risk of failure and harm in processes as well as to identify the most important areas for process improvements. FMEA has been used by hospitals in a variety of Institute for Healthcare Improvement programs in the US, including Idealized Design of Medication Systems, patient safety collaboratives, and the Patient Safety Summit. ${ }^{9}$ It involves reviewing as many components, assemblies, and subsystems as possible to identify failure modes and their causes and effects. For each component, the failure modes and their resulting effects on the rest of the system are recorded in a specific FMEA worksheet. FMEA includes the review of the following:

1. Steps in the process

2. Failure modes (What could go wrong?)

3. Failure causes (Why would the failure happen?)

4. Failure effects (What would be the consequences of each failure?)

Within this approach, the critical treatment path is followed and risk classification for each individual step is estimated (Figure 1).

The method is explained in detail in Ponzetti et al. ${ }^{10}$

In the first instance, four centers in two regions (EmiliaRomagna and Lombardia) were identified in order to run a pilot study phase analyzing the feasibility. The regions were selected with one breast cancer and one hematologic center per region. The pilot study was successful and demonstrated trends toward a benefit of the subcutaneous therapy. ${ }^{11}$ After the successful execution of the pilot study, 19 centers in six Italian regions were recruited to participate in the study. 
The two largest regions participating in the study were Emilia-Romagna and Lazio (Table 1).

Information were collected for five patients per participating center using a validated questionnaire. Within that survey the current information on the administration of trastuzumab in breast cancer and rituximab in NHL were collected and compared against the expected results for the subcutaneous therapy (Figure 2). The rationale for the sample size per center was based on the average patient records per week. Base assumptions were as follows: the health care processes are consistent and well-defined between the centers, and the sample did not have the purpose of being statistically

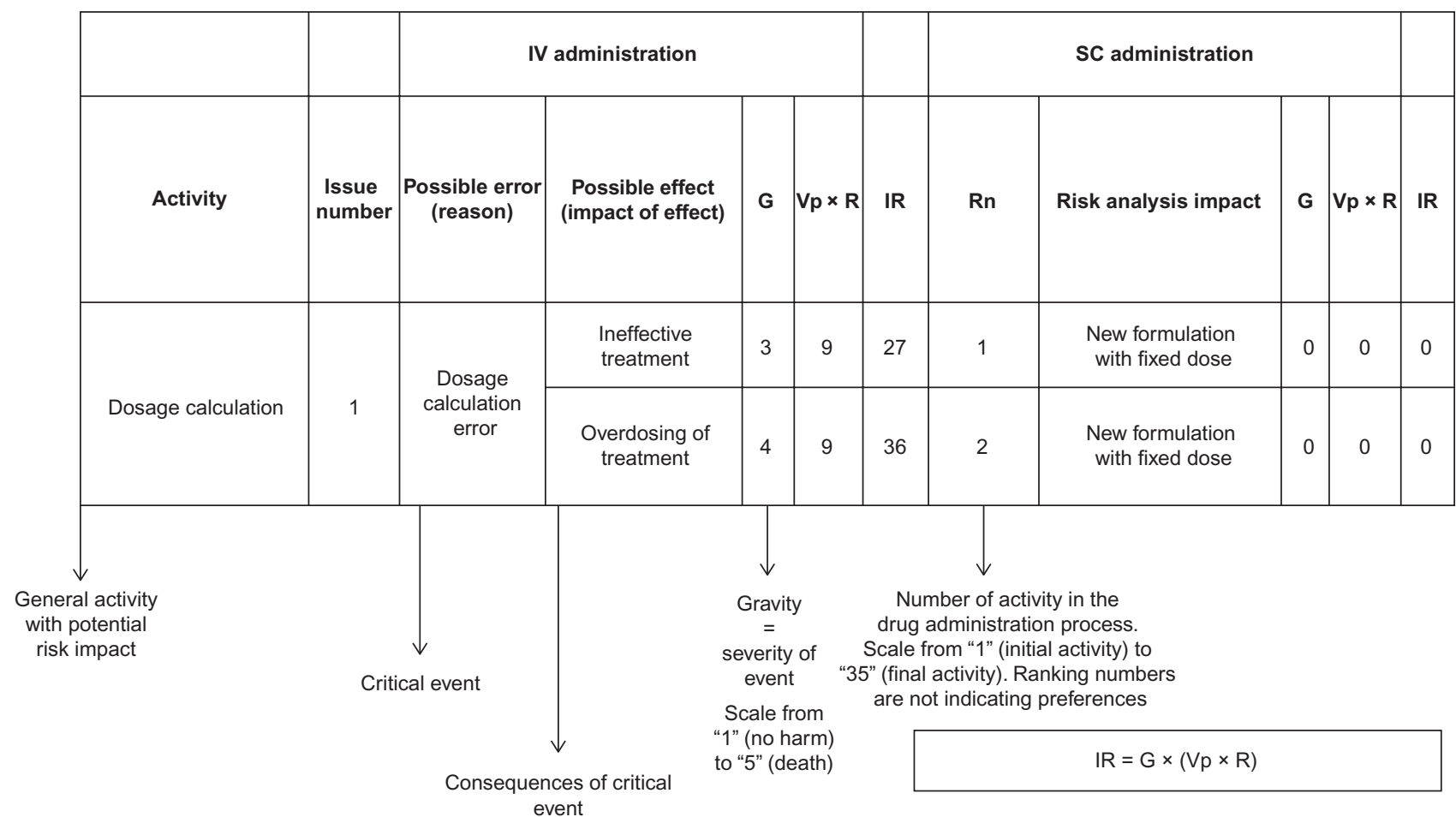

Figure I FMEA calculation method illustrated with the risk on dosage error.

Abbreviations: IV, intravenous; SC, subcutaneous; G, gravity/severity of event; Vp, probability of event; R, reliability of event; IR: risk index; Rn, rank; FMEA, Failure Mode and Effect Analysis.

Table I Overview of the 19 centers in the six Italian regions participating in the study

\begin{tabular}{|c|c|c|c|}
\hline Region & Center & Hematology & Oncology \\
\hline \multirow[t]{4}{*}{ Emilia-Romagna } & Istituto Scientifico Romagnolo per lo Studio e la Cura dei Tumori, Meldola & $\mathbf{X}$ & $\mathbf{X}$ \\
\hline & Ospedale Santa Maria delle Croci, Ravenna & $\mathbf{X}$ & $\mathbf{X}$ \\
\hline & Azienda Ospedaliero-Universitaria di Parma, Ospedale Maggiore & $\mathbf{X}$ & $\mathbf{X}$ \\
\hline & Nuovo Ospedale S. Anna, Cona, Ferrara & $\mathbf{x}$ & $\mathbf{x}$ \\
\hline \multirow[t]{6}{*}{ Lazio } & Università Cattolica del Sacro Cuore, Policlinico A. Gemelli & $\mathbf{X}$ & $\mathbf{x}$ \\
\hline & Istituto Nazionale Tumori Regina Elena Irccs-Ifo & $\mathbf{x}$ & $\mathbf{x}$ \\
\hline & Azienda Ospedaliera Universitaria Policlinico Tor Vergata, Roma & $\mathbf{X}$ & $\mathbf{X}$ \\
\hline & Ospedale di Ronciglione, Viterbo & $\mathbf{x}$ & \\
\hline & Policlinico Universitario Campus Roma & $\mathbf{X}$ & \\
\hline & Ospedale Santa Maria Goretti Latina & $\mathbf{X}$ & $\mathbf{X}$ \\
\hline \multirow[t]{4}{*}{ Liguria } & Azienda Ospedaliera Universitaria San Martino-IST, Genova & $\mathbf{X}$ & $\mathbf{X}$ \\
\hline & ULSS I Imperiese Ospedale Bussana San Remo & $\mathbf{X}$ & $\mathbf{X}$ \\
\hline & ULSS 3 Genovese Villa Scassi & $\mathbf{X}$ & \\
\hline & Ospedale Galliera, Genoa & & $\mathbf{X}$ \\
\hline \multirow[t]{2}{*}{ Piemont } & Ospedale degli Infermi di Biella & $\mathbf{X}$ & $\mathbf{X}$ \\
\hline & Azienda Ospedaliera Universitaria Ospedale Maggiore della carità di Novara & & $\mathbf{X}$ \\
\hline \multirow[t]{2}{*}{ Toscana } & Azienda Ospedaliero-Universitaria Careggi (AOUC) & $\mathbf{X}$ & $\mathbf{X}$ \\
\hline & Azienda Ospedaliero Universitaria Pisana & $\mathbf{X}$ & $\mathbf{X}$ \\
\hline Umbria & Azienda Ospedaliera di Perugia-Ospedale Santa Maria della Misericordia & $\mathbf{x}$ & $\mathbf{x}$ \\
\hline
\end{tabular}




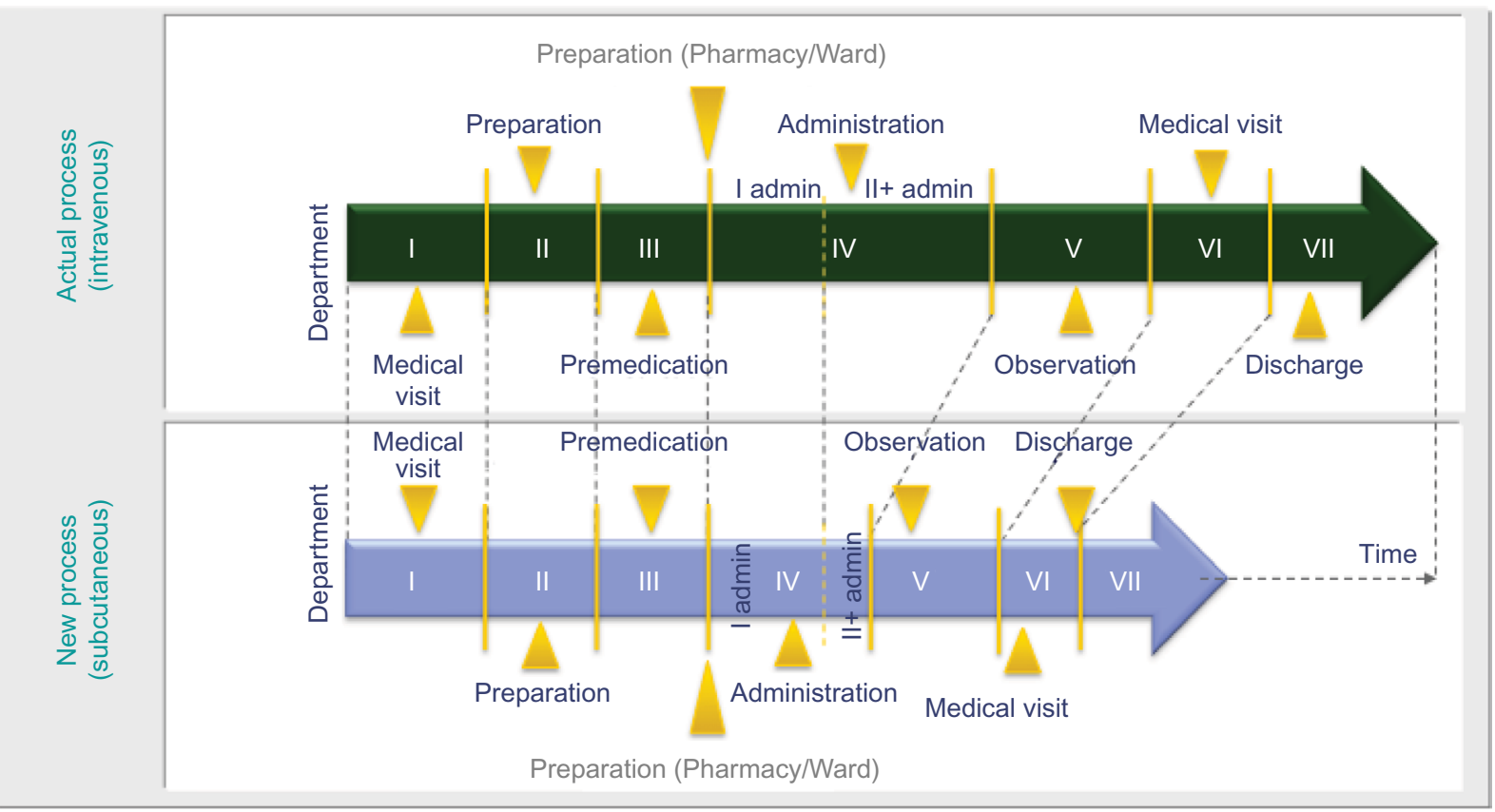

Figure 2 The theoretical model to analyze the subcutaneous versus intravenous therapy benefits in a real-life setting in Italy. Notes: I admin, first administration; II + admin, therapy administration after the first administration.

Table 2 Size differences in participating centers

\begin{tabular}{lll}
\hline $\begin{array}{l}\text { Differentiation in } \\
\text { treatment centers } \\
\text { according to size }\end{array}$ & $\begin{array}{l}\text { Number of } \\
\text { hematology } \\
\text { centers }\end{array}$ & $\begin{array}{l}\text { Number of } \\
\text { oncology centers }\end{array}$ \\
\hline $\begin{array}{l}\text { Large center (>100 } \\
\text { patients annually) }\end{array}$ & $9(53 \%)$ & $4(24 \%)$ \\
$\begin{array}{l}\text { Medium-sized center } \\
\text { (between } 99 \text { and } 50\end{array}$ & $4(24 \%)$ & $5(29 \%)$ \\
patients annually) & & \\
$\begin{array}{l}\text { Small center }(<50 \\
\text { patients annually) }\end{array}$ & $4(24 \%)$ & $7(41 \%)$ \\
Total & $17(100 \%)$ & $16(100 \%)$ \\
\hline
\end{tabular}

Abbreviation: NHL, non-Hodgkin's lymphoma.

representative but rather to provide an overview of operating modes consolidated. The route of administration was discussed and aligned with the participating centers in order to capture all relevant parts of the therapy.

Besides the location, the annual number of treated patients could also have an impact on the interpretability of the study. As it can been seen in Table 2, 50\% of participating hematology centers treat $>100$ patients annually and are defined as being large centers. The proportion of medium and small-sized centers is quite evenly distributed with $24 \%$ and $29 \%$, respectively. For the oncology centers with a focus on breast cancer patients, there are approximately $41 \%$ small study centers compared to $29 \%$ medium-sized and $24 \%$ large centers.
For the rituximab and trastuzumab administration, 35 different risk steps were identified and assessed. The summary pathway with detailed levels is available in Figure 3. A detailed description of all the 35 areas are available in Table S1.

After the identification of the risk levels for intravenous and subcutaneous administration, the monetary quantification of the insurance premium reduction was calculated as follows: it was assumed that risk classes of three or lower would not require separate insurance or would not have an impact on the insurance premiums for a hospital. For risk level 4, the following scenario was assumed: a female patient, 50 years of age, who receives a biologic therapy against her breast cancer would need to visit the hospital once a week. It is assumed that a treatment or administration error with a risk level 4 would impact the patient by a permanent invalidity of $40 \%$. Based on a decision by the Milan court, ${ }^{12}$ a so-called table 2013 was published that shows that such a permanent disability would have a cost impact of minimum $€ 234,371$ Such a risk would need to be insured additionally by a special insurance for each hospital. The exact premiums could not be calculated; however, it could be assumed that a reduction in the likelihood of such a monetary impact would also have a proportional impact on the insurance premiums. No ethical approval was required as no actual patients were involved. 


\begin{tabular}{|c|c|}
\hline Phase & Activity \\
\hline \multirow{2}{*}{ Therapy prescription } & Dosage calculation \\
\hline & $\begin{array}{l}\text { Application to the pharmacy to prepare the } \\
\text { pharmacotherapy }\end{array}$ \\
\hline \multirow{3}{*}{$\begin{array}{l}\text { Preparation of the } \\
\text { pharmaceutical therapy }\end{array}$} & Pick up of therapy at the hospital pharmacy \\
\hline & Therapy set up \\
\hline & Package labeling \\
\hline \multirow{11}{*}{$\begin{array}{l}\text { Administration of the } \\
\text { pharmacotherapy }\end{array}$} & Patient identification \\
\hline & Receipt of the pharmacotherapy from pharmacy \\
\hline & Verification of the corresponding patient \\
\hline & Preparation of the venous therapy \\
\hline & Infusion set up \\
\hline & Predisposition of the infusion pump \\
\hline & Infusion of secondary scheme \\
\hline & Infusion control with respect to velocity \\
\hline & $\begin{array}{l}\text { Needle/syringe control and regulation of venous } \\
\text { access }\end{array}$ \\
\hline & Intervention in case of pump issues \\
\hline & Patient intervention call \\
\hline Therapy/treatment & Medication/treatment via infusion \\
\hline
\end{tabular}

A Identification of:

-Possible events

-Possible

consequences

B

Individual level of:

-Severity

-Likelihood

-Detectability

Definition of RI

Confrontation of

D ex-ante and ex-post

risk with and

without the new

formulation

Figure 3 Therapy phases and its activities based on the defined FMEA critical pathway.

Abbreviations: FMEA, Failure Mode and Effect Analysis; RI, risk index.

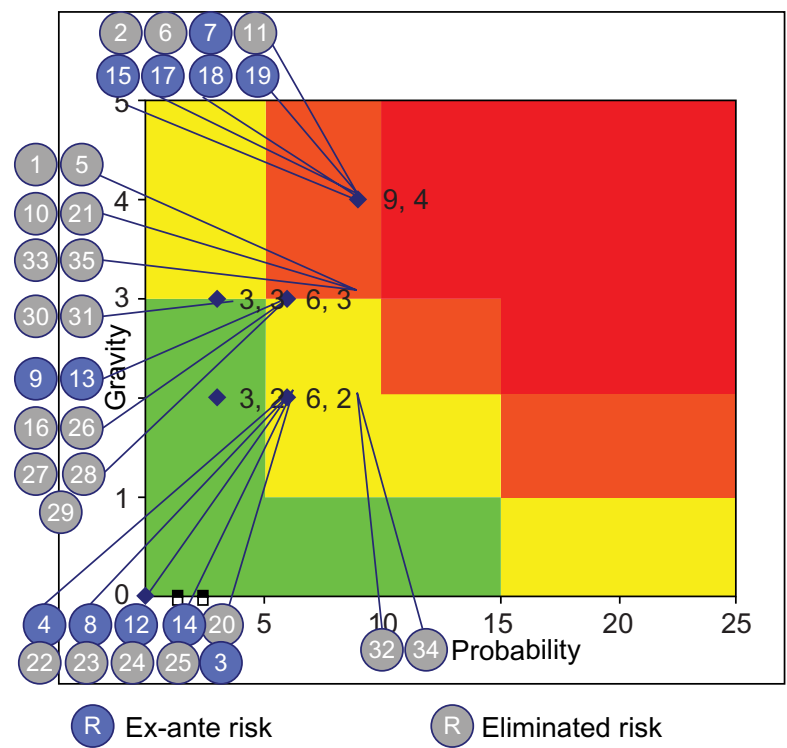

Figure 4 Risk levels in a risk matrix for the intravenous administration of rituximab in NHL and trastuzumab in breast cancer in Italy, in comparison to the subcutaneous therapy.

Note: Description of risk classes are available on request from the authors. Abbreviation: NHL, non-Hodgkin's lymphoma.

\section{Results}

When the risk classes are followed and calculated, eight areas were identified to be high risk for the administration of an intravenous therapy in hematology or oncology, 13 areas would be defined as having a median risk, and 14 areas would be classified as having a low risk classification (Figure 4). The eight high-risk areas identified are as follows (numbers in brackets correspond to the respective FMEA rankings in Table S1):
1. Overdosing of treatment due to dosage calculation error (2)

2. Overdosing of treatment due to wrong/missing prescription check (6)

3. Wrong patient treated due to wrong/missing prescription check (7)

4. Overdosing due to wrong therapy preparation (11)

5. Treatment to another (wrong) patient due to wrong drug arrival (15)

6. Treatment to another (wrong) patient due to wrong patient identification (17)

7. Treatment to another (wrong) patient due to missing check of patient and drug bag (label) (18)

8. Treatment to another (wrong) patient due to wrong check of patient and drug bag (label) (19)

When the new subcutaneous formulation would be applied, 23 different risk levels could be completely eliminated, which is a $65 \%$ reduction in risk levels (Figure $3 \mathrm{~B}$ ). Including those eliminations are the following important and high-risk classes:

1. Dose calculation

2. Preparation and package labeling

3. Preparation of the access to the vein

4. Pump infusion preparation and infusion monitoring

Including the infusion preparation and application of the infusion might also lead to the reduction of patientrelevant events such as infections and other infusion-related issues. Furthermore, the following risk classes might possibly be moved from high risk or medium risk to the lower 
risk and lower likelihood of occurrence by changing the administration route to subcutaneous:

1. Wrong preparation

2. Missing labeling

3. Wrong labeling

4. Wrong check of corresponding patient bag

The overall risk level for the intravenous administration was estimated to be 756 (ex-ante) and could be reduced by $70 \%$ to a risk score of 225 (ex-post). Such an impact is patient relevant and also has a major impact on the insurance premiums being paid for such risks or the accruals a hospital needs to take for the potential financial implications. As the actual premiums could not be taken into account, an approximation based on the potential compensation for the harm was utilized. The likelihood for such a potential reduction is based on the lower risk index and lower risk potentials with the subcutaneous administration.

\section{Discussion}

Based on the authors' knowledge, this analysis is one of the first published articles for the Italian health care setting evaluating the potential impact of a new subcutaneous formulation in hematology and oncology on the risk quantification. The results show a relevant decrease in the risk index and also a potential relevant financial impact with respect to insurance premiums being charged for each hospital or accruals to be taken to compensate the potential financial risks. In the current economic situation, hospitals in Europe and especially in Italy are under financial and health care quality pressure. These results have a large relevance in terms of therapy quality assurance from a patient's perspective linked with potential cost savings in terms of insurance premiums. Furthermore, the underlying analysis shows potential for cost and resource savings in hospitals due to subcutaneous administration of trastuzumab and rituximab.

The underlying analysis might be criticized based on the applied method. The analysis was done with a comparison of the actual situation with the intravenous therapy and was compared to the theoretical savings of a subcutaneous therapy. The theoretical risk reduction needs to be taken into account with the already existing risk mitigation strategies by hospitals and could hence be overestimated. Furthermore, the real-life impact would need to be captured in a direct clinical study. Finally, the impact on the insurance premiums for hospitals due to treatment errors would need further research. Additionally, the number of centers were acceptable for such a research; however, two out of 17 centers contributed $>50 \%$ of patients observed for the analysis in NHL and four out of 16 centers in breast cancer contributed $50 \%$ of participating patients. This center bias will most likely have an impact on the results. When sensitivity analyses were run, the results were consistent across the different regions.

\section{Conclusion}

The availability and use of subcutaneous administration for oncologic or hematologic therapies might lower the risk of administration and treatment errors for patients and hence could indirectly have a positive financial impact for hospitals through lower insurance premiums against such risks. The availability of a subcutaneous version of rituximab and trastuzumab in the approved indications offers the availability of the current standard of care with a reduced risk of treatment errors.

\section{Disclosure}

$\mathrm{MC}$ and MF are employees of EmmEffe and received funding from Roche S.p.a. for executing the research. SE is an employee of Roche S.p.a. SW received funding from Roche S.p.a. for interpretation, analysis, and writing of the research. The authors report no other conflicts of interest in this work.

\section{References}

1. European Medicine Agency. Trastuzumab (Herceptin). Summary of Product Characteristics. 2014.

2. Cardoso F, Costa A, Norton L, et al. ESO-ESMO 2nd international consensus guidelines for advanced breast cancer (ABC2). Ann Oncol. 2014;25(10):1871-1888.

3. Cardoso F, Costa A, Norton L, et al. ESO-ESMO 2nd international consensus guidelines for advanced breast cancer (ABC2). Breast. 2014;23(5):489-502.

4. Widakovich C, de Castro G Jr, de Azambuja E, Dinh P, Aawda A. Review: side effects of approved molecular targeted therapies in solid cancers. Oncologist. 2007;12:1443-1455.

5. Agenzia Italiana del Farmaco (AIFA). Application of the new European legislation on pharmacovigilance changed with the adoption in 2010 of EU Regulation 1235/2010 and Directive 2010/84 / EU. 2012. Available from: http://ec.europa.eu/health/files/eudralex/vol-1/dir_2010_84/ dir_2010_84_en.pdf. Accessed April 21, 2016.

6. Carleton BC, Smith MA. Drug safety: side effects and mistakes or adverse reactions and deadly errors? BCMJ. 2006;48(7):329-333.

7 European Medicine Agency. Rituximab (Mabthera). Summary of Product Characteristics. 2015.

8. Stamatitis DH. FMEA from theory to execution. Failure Mode and Effect Analysis. 2nd ed. Milwaukee (WI): American Society for Quality, Quality Press: 2003.

9. Institute for Health Care Improvement: Failure Modes and Effects Analysis (FMEA) Tool. Available from: http://www.ihi.org/resources/Pages/Tools/ FailureModesandEffectsAnalysisTool.aspx. Accessed July 10, 2015.

10 Ponzetti C, Canciani M, Farina M, Era S, Walzer S. Potential resource and cost saving analysis of subcutaneous versus intravenous administration for rituximab in non-Hodgkin's lymphoma and for trastuzumab in breast cancer in 17 Italian hospitals based on a systematic survey. ClinicoEconomics and Outcomes Research. In press 2016. Available from: http://www.healthcommunity.it/wp-content/uploads/2015/11/ Educational.pdf. Accessed April 21, 2016.

11. Farina M, Canciani M, Ponzetti C. Il ruolo di formulazioni sottocutanee dei farmaci biologici nell'ottimazazione dei processi di cura in oncologiga ed ematologia. Frammenti Educational. 2014;Suppl n.32 anno 8.

12. Tribunale di Milano. 2013. Liquidazione del danno non patrimoniale Tabelle 2013. 


\section{Supplementary material}

Table SI Overview of all FMEA activities included in the analysis and its respective $\mathrm{Rn}$

\begin{tabular}{lll}
\hline Therapy stage & Activity & Possible error (reaso \\
\hline Therapy prescription & Dosage calculation & Dosage calculation erro \\
& $\begin{array}{l}\text { Prescription hand over to pharmacy } \\
\text { preparing the therapy } \\
\text { Prescription check }\end{array}$ & Sending delay \\
Pharmacy preparation & Wrong/missing check
\end{tabular}

\begin{tabular}{|c|c|c|c|c|}
\hline & & & Wrong patient treated & 7 \\
\hline & Drug taking & Missing/wrong drug taking & Treatment delay & 8 \\
\hline & & Wrong drug taking & Wrong treatment & 9 \\
\hline & Drug preparation & Wrong preparation & Ineffective treatment & 10 \\
\hline & & & Treatment overdosing & II \\
\hline & Bag labeling & Missing labeling & Delayed treatment & 12 \\
\hline & & Wrong labeling & Wrong treatment & 13 \\
\hline Pharmacy administration & Pharmacy receiving actual drug after & Late drug arrival & Delayed treatment & 14 \\
\hline & preparation & $\begin{array}{l}\text { Wrong drug arrival (to the wrong } \\
\text { patient) }\end{array}$ & Treatment to another patient & 15 \\
\hline & & $\begin{array}{l}\text { Wrong drug arrival (wrong } \\
\text { preparation) }\end{array}$ & Wrong treatment & 16 \\
\hline & Patient identification & Wrong identification & Treatment to another patient & 17 \\
\hline & Check correspondence patient/bag & Missing check & Treatment to another patient & 18 \\
\hline & & Wrong check & Treatment to another patient & 19 \\
\hline & Venous access & No venous access & Missing treatment & 20 \\
\hline & & Wrong venous access & Wrong treatment & 21 \\
\hline & Infusion via preparation & Missing preparation infusion & Delayed treatment & 22 \\
\hline & & Wrong preparation infusion & Delayed treatment & 23 \\
\hline & & & Missing treatment (not functioning) & 24 \\
\hline & Preparation infusion pump & Missing preparation infusion pump & Treatment delay & 25 \\
\hline & & Wrong preparation infusion pump & $\begin{array}{l}\text { Wrong treatment (due to infusion } \\
\text { speed) }\end{array}$ & 26 \\
\hline & $\begin{array}{l}\text { Second scheme infusion } \\
\text { administration }\end{array}$ & Wrong reading & Wrong treatment & 27 \\
\hline & Infusion speed check & Wrong/missing speed check & Wrong treatment & 28 \\
\hline & Patency check and regular vein check & Wrong/missing speed check & $\begin{array}{l}\text { Wrong administration (patency } \\
\text { and extraversion) }\end{array}$ & 29 \\
\hline & Work on the pump to alarm or alert & Missing intervention & Wrong treatment & 30 \\
\hline & the patient & Intervention delay & Wrong treatment & 31 \\
\hline End of treatment & Medication/treatment via infusion & Missing medication/treatment & Infection & 32 \\
\hline & utilization & & Occlusion venous access & 33 \\
\hline & & Wrong medication & Infection & 34 \\
\hline & & & Occlusion venous access & 35 \\
\hline
\end{tabular}

Abbreviations: FMEA, Failure Mode and Effect Analysis; Rn, rank.

ClinicoEconomics and Outcomes Research

\section{Publish your work in this journal}

ClinicoEconomics and Outcomes Research is an international, peerreviewed open-access journal focusing on health technology assessment, pharmacoeconomics and outcomes research in the areas of diagnosis, medical devices, and clinical, surgical and pharmacological intervention. The economic impact of health policy and health systems organization also constitute important areas of coverage. The manuscript management system is completely online and includes a very quick and fair peer-review system, which is all easy to use. Visit http://www.dovepress.com/testimonials.php to read real quotes from published authors. 